\title{
Analyse und Anpassung ausgewählter Optimierungsverfahren für den Digitalen Zwilling von Verdichteranlagen
}

\author{
R. Thiel ${ }^{1}$, J. Jäkel ${ }^{1}$, K. Schleifer ${ }^{2}$, R. Schulze ${ }^{2}$
}

\section{Zusammenfassung}

Digitale Zwillinge werden in der Betriebsphase einer Maschine oder Anlage für verschiedene Fragestellungen angewendet, bspw. für die Zustandserkennung. Voraussetzung dafür ist, dass der Digitale Zwilling über Modelle des Anlagenverhaltens verfügt, die verschiedene Zustände, einschließlich von Fehlerzustände, adäquat beschreiben. Ein wesentlicher Aspekt dabei ist die Identifikation der Modellparameter. Dieser Beitrag dient der Analyse und Anpassung ausgewählter Optimierungsmethoden (gradientenbasierter Verfahren und ein Partikelschwarmoptimierer) am Beispiel von Modellen für Kompressoren und Turbinenanlagen. Dazu wird mit einem analytischen Ansatz ein Modell einer realen Kompressoranlage erstellt. Um die Qualität der Optimierungsergebnisse vergleichen zu können, wird eine Fehlermetrik eingeführt. Das Verhalten der Optimierer wird unter den folgenden realistischen Fehlerszenarien ermittelt: Fehler in den Modellparametern, Rauschen, initialer Abstand vom Optimum, fehlerhaft Messstellen (ohne und mit Detektion). Zur numerischen Bewertung der Identifizierbarkeit werden zwei Kriterien eingeführt.

\section{Stichwörter}

Modellbildung, Simulationsmodell, Parameteridentifikation, Digitaler Zwilling, Turboverdichter, Smart Monitoring

\section{Einführung}

Turbinen- und Verdichteranlagen unterliegen einer kontinuierlichen Alterung und Abnutzung, die durch einfache Messung von Prozessgrößen nur schwer zu überwachen ist. Die modellbasierte Überwachung des Anlagenzustandes soll hier Abhilfe schaffen. Dazu werden auf der Basis des Digitalen Zwillings bzw. des darin enthaltenen Anlagenmodells durch einen Vergleich zwischen messtechnisch erfassten Größen der Anlage und den simulierten Größen des Anlagenmodells Informationen über den konkreten Anlagenzustand parallel zum Betrieb gewonnen. Darüber hinaus ist es auch möglich, nicht gemessene Größen zu ermitteln. Für die genannten Aufgabenstellungen ist eine kontinuierliche Anpassung der Modelle eines Digitalen Zwillings auf Grundlage der realen Messdaten notwendig und bedarf eines hohen Automatisierungsgrads bezüglich der Datenfilterung, -validierung und der Bestimmung von Betriebszuständen.

Die komplexe numerische Herausforderung der Parameteroptimierung auf Modellebene im Digitalen Zwilling wird mithilfe von nichtlinearen Optimierungsverfahren gelöst [1, 2]. In [3, 4] wird die Effizienz solcher Verfahren anhand der Parameterbestimmung von Modellen elektrischer Maschinen bzw. von Robotern aufgezeigt. Eine weitere Möglichkeit besteht in der Verwendung metaheuristischen Suchverfahren, wie genetischen Algorithmen, die sich aufgrund ihrer hohen Flexibilität besonders für

$1 \quad$ HTWK Leipzig, Fakultät für Ingenieurwissenschaften, Leipzig, Deutschland

2 Avicomp Controls GmbH, Leipzig, Deutschland 
komplexe Parameteroptimierungsaufgaben eignen. Speziell bei stationären Betriebszuständen haben sich derartige Verfahren bewährt $[4,5]$.

Ziel des Beitrages ist die vergleichende Analyse von Optimierungsverfahren für die Parameteridentifikation anhand eines Modells eines Turboverdichters. Hierbei interessieren insbesondere die Güte der Optimierunsgverfahren, ihre Robustheit bezüglich der Änderungen in den Messdaten, die Abhängigkeit der Ergebnisse vom Signal-Rausch-Verhältnis und den Startwerten, darüber hinaus der Rechenzeitbedarf. Ein weiteres analysiertes Problem ist die Identifizierbarkeit.

Im nächsten Abschnitt werden neben dem Modell die verwendeten Zielfunktionen und entwickelten Kriterien zur numerischen Bewertung der Identifizierbarkeit beschrieben, In Abschnitt 3 wird die Versuchsdurchführung, in Abschnitt 4 die Ergebnisse dargestellt. Abschließend werden diese in Abschnitt 5 ausgewertet.

\section{Methoden}

\subsection{Verdichtermodell}

Die wichtigste Größe eines Verdichtermodells ist die Druckänderung, welche durch

$$
p_{2}=p_{1}\left(\frac{\frac{n_{p}-1}{n_{p}} \cdot Y_{p}}{R_{S} T_{1} Z_{1}\left(p_{1}, T_{1}\right)}+1\right)^{\frac{n_{p}}{n_{p}-1}}
$$

definiert ist. Größen mit dem Index 1 markieren die Eingangsgrößen, mit dem Index 2 die Ausgangsgrößen. Dabei sind $p$ der Druck, $T$ die Temperatur, $R_{S}$ die spezifische Gaskonstante, $Z$ der Kompressibiltätsfaktor, $n_{p}$ der Druckexponent und $Y_{p}$ die polytrope Förderhöhe. Die polytrope Förderhöhe berechnet sich über

$$
Y_{P}=f(\dot{V}, N)
$$

mit der Verdichterdrehzahl $N$ und dem Volumenstrom $\dot{V}$, welcher sich aus

$$
\dot{V}=\frac{\dot{m}}{\rho}
$$

dem Massenfluss $\dot{m}$ und der Gasdichte $\rho$ ergibt.

Für jeden Verdichter wird ein Kennfeld für den Zusammenhang zwischen polytroper Förderhöhe, Drehzahl und Volumenstrom vom Hersteller mitgeliefert. Dieses bildet die Basis zur Berechnung im Digitalen Zwilling. Die Approximation dieses Kennfeldes wird im folgenden Abschnitt erläutert. Da sich das Kennfeld während der Lebenszeit ändert, wird (2) um die beiden KennfeldVerschiebungsparameter $P_{a x}$ und $P_{a y}$ wie folgt erweitert

$$
Y_{P}=f\left(\dot{V}+P_{a x}, N\right)+P_{a y} .
$$

Die Temperaturänderung durch den Verdichter wird durch

$$
T_{2}=T_{1}\left(\frac{p_{2}}{p_{1}}\right)^{\frac{n_{T}-1}{n_{T}}}
$$

beschrieben, hierbei ist $n_{T}$ der Temperaturexponent. Die Gleichungen (1) und (5) werden in [7] hergeleitet. Der Druckverlust im Ein- und Ausgangsrohr wird über die Darcy-Weisbach-Gleichung

$$
\Delta p=f(p, T, \dot{m})=\lambda \frac{8 L}{\rho \pi^{2} D^{5}}|\dot{m}| \cdot \dot{m}
$$

berechnet. Dabei ist $L$ die Rohrlänge, $D$ der Rohrdurchmesser, $\lambda$ der Rohrreibungsfaktor und $\rho$ die Gasdichte. Die Dichte $\rho$ wird über

$$
\rho=\frac{p}{R_{S} Z T} \text { mit } R_{S}=\frac{R}{M}
$$

berechnet. Im Rohrreibungsfaktor $\lambda$ werden alle unbekannten Einflüsse wie Druckänderung durch Rohrbögen und Rohrverjüngung durch Ablagerung zusammengefasst. 


\subsection{Kennfeld-Modell}

Für jeden Verdichter wird bei der Inbetriebnahme ein Kennfeld vom Hersteller mitgeliefert. Für die Optimierung im Digitalen Zwilling werden drei verschiedene Verfahren zur Kennfeldapproximation untersucht. Das Standardverfahren zur Approximation ist die Interpolation durch ein Polynom dritten Grades

$$
Y_{P}(\dot{V}, N)=p_{00}+p_{10} \dot{V}+p_{01} N+p_{11} \dot{V} N+p_{20} \dot{V}^{2}+p_{02} N^{2}+p_{21} \dot{V}^{2} N+p_{12} \dot{V} N^{2}+p_{30} \dot{V}^{3}+p_{03} N^{3} .
$$

Dabei werden die Parameter $p$ so gewählt, dass der quadratische Restfehler zwischen Kennfeld und Polynom minimal ist. Als weitere Approximationsmethoden wird ein einfaches Interpolationsverfahren gewählt, bei welchem ausgewählte feste Drehzahlkennlinien $N_{c}$ mittels

$$
Y_{P}\left(\dot{V}, N_{c}\right)=a \mathrm{e}^{b x}+c \mathrm{e}^{d x}
$$

approximiert werden. Für Werte zwischen den festen Drehzahlen wird linear interpoliert. Das dritte Verfahren verwendet ebenfalls ein Polynom. Dabei wird auf die Gleichung (2) vorher die Dimensionsanalyse nach [10] angewendet. Damit wird der Bereich, der im Kennfeld approximiert werden muss, kleiner, siehe Abb. 10 im Abschnitt Auswertung. Aus Gleichung (4) wird damit zu

$$
Y_{P}=f\left(\frac{\dot{V}}{\rho N D^{3}}+P_{a x}, N\right) N^{2} D^{2}+P_{a y} .
$$

Eine ausführliche Beschreibung der Dimensionsanalyse ist in [7] enthalten.

\subsection{Variablen}

Zusammengefasst enthält das Modell für die Verwendung im Digitalen Zwilling die in Tab. 1 aufgeführten Variablen und Parameter.

Tab. 1: Variablen- und Parameterübersicht

\begin{tabular}{llcc}
\hline & Name & Symbol & Einheit \\
\hline \hline \multirow{3}{*}{ Messgrößen } & Ein- und Ausgangsdruck & $p_{1}, p_{2}$ & $\mathrm{bar}$ \\
& Ein- und Ausgangstemperatur & $T_{1}, T_{2}$ & $\mathrm{~K}$ \\
& Massenfluss & $\dot{m}$ & $\mathrm{~kg} / \mathrm{s}$ \\
& Drehzahl & $N$ & $1 / \mathrm{s}$ \\
\hline \multirow{3}{*}{ Konstanten } & Rohrlänge & $L$ & $\mathrm{~m}$ \\
& Rohrdurchmesser & $D$ & $\mathrm{~m}$ \\
& Gaskonstante & $R$ & $\frac{\mathrm{kg} \mathrm{m}^{2}}{\mathrm{~s}^{2} \mathrm{~mol} \mathrm{~K}^{\mathrm{K}}}, \frac{\mathrm{J}}{\mathrm{mol} \mathrm{K}}$ \\
& spezifische Gaskonstante & $R_{S}$ & $\frac{\mathrm{J}}{\mathrm{kg} \mathrm{K}}$ \\
\hline \multirow{3}{*}{ Parameter (Optimierungsvariablen) } & Rohrreibungsfaktor & $\lambda_{1}, \lambda_{2}$ & \\
& Druckexponent & $n_{p}$ & \\
& Kemperaturexponent & $n_{T}$ & \\
& Kennfeld-Verschiebungsparameter $x$ & $P_{a x}$ & $\frac{\mathrm{m}^{3}}{\mathrm{~s}}$ \\
& Kennfeld-Verschiebungsparameter $y$ & $P_{a y}$ & $\mathrm{~m}$ \\
\hline
\end{tabular}




\subsection{Optimierungsverfahren}

Zur Bestimmung der unbekannten Parameter werden Optimierungsverfahren und Messdaten, die an Arbeitspunkten aufgenommen werden, verwendet. Da der Digitale Zwilling mittels Matlab/Simulink erstellt wurde, werden nur Optmimierungsverfahren betrachtet, die in Matlab einschließlich der $O p$ timization Toolbox implementiert sind. Als Referenz wird der Optimierer fmincon mit dem Algorithmus interior-point [8] verwendet. Als Zielfunktion wird der kleinste quadratische Fehler

$$
\min _{p}\|F(\hat{x}, p)-\hat{y}\|_{2}^{2}=\min _{p} \sum_{i}\left(F_{i}\left(\hat{x}_{i}, p\right)-\hat{y}_{i}\right)^{2}
$$

verwendet. Dabei ist $F$ das Modell des Verdichters, $p$ der Parametervektor und $\hat{x}, \hat{y}$ sind die Messdaten, $i$ indiziert den Arbeitspunkt. Zusätzlich werden für die Parameterwerte Schranken vorgegeben. Verglichen wird fmincon mit den Optimierern fminimax und lsqcurvefit mit den Algorithmen trustregion-reflective und levenberg-marquardt sowie einem Partikelschwarmoptimierer. Die Zielfunktion ist bei allen identisch mit Ausnahme von fminimax mit der Zielfunktion

$$
\min _{p} \max _{i}\left(F_{i}\left(\hat{x}_{i}, p\right)-\hat{y}_{i}\right)^{2} \text {. }
$$

Bei diesem wird nur der Wert mit der größten Abweichung optimiert.

\subsection{Zielfunktion für robuste Optimierung}

Ein Problem aus der Praxis ist der überproportionale Einfluss von gruppierten Arbeitspunkten. Daher wird die Gleichung (11) um eine Abstandswichtung erweitert. Dafür werden die euklidischen Abstände im Eingangsraum, welcher von den Eingangsmessdaten Druck, Temperatur und Massenfluss aufgespannt wird, verwendet. Diese werden vorher auf den Referenz-Arbeitspunkt im Kennfeld normiert (vgl. 3.3). Die Distanzmessung für jeden Arbeitspunkt $i$ berechnet sich zu

$$
d_{i}=\frac{1}{N_{A P}} \sum_{j=1}^{N_{A P}} \sqrt{\left(\dot{m}_{i}-\dot{m}_{j}\right)^{2}+\left(p_{i}-p_{j}\right)^{2}+\left(T_{i}-T_{j}\right)^{2}},
$$

wobei $N_{A P}$ die Anzahl der Arbeitspunkte ist. Die sich damit ergebenden Zielfunktion lautet

$$
\min _{x} \sum d_{i}\left(F\left(x, \hat{x}_{i}\right)-\hat{y}_{i}\right)^{2} .
$$

\subsection{Qualitätskriterium für Optimierungsparameter}

Ein grundlegendes Problem bei Optimierungsaufgaben ist die Abhängigkeit von Optimierungsvariablen. In der Anwendung bedeutet das, dass die Änderung von zwei oder mehreren Optimierungsparametern den gleichen Einfluss auf das Ergebnis haben und somit kein eindeutiges Optimum existiert. Solche Abhängigkeiten in komplexen Modellen aufzufinden und zu eliminieren, ist nicht trivial. Bei der Parameteridentifikation spricht man auch von (struktureller) Identifizierbarkeit (vgl. [9]). Um diese Äbhängigkeiten und ihren Einfluss bewerten zu können, werden zwei Qualitätskriterien $A Z_{1}$ und $A Z_{2}$ eingeführt. Diese beschreiben den gemeinsamen Einfluss zweier Modellparameter (Optimierungsvariablen) auf die gewählte Ausgangsgröße. Die Abhängigkeit wird von Null (keine Abhängigkeit) bis Eins (hohe Abhängigkeit) angegeben. Dabei gibt $A Z_{1}$ an, wie ähnlich zwei Variablen dieselbe Ausgangsgröße verändern, und $A Z_{2}$, ob mehrere Arbeitspunkte das Ergebnis verbessern können. Diese Kriterien dienen dazu, den Parametersuchraum und damit das Optimierungsproblem zu vereinfachen und mehrfache gleichwertige Optima zu eliminieren. Hierfür wird zunächst eine Hilfsgröße eingeführt

$$
a z=\left|1-\left(\operatorname{atan} 2\left(\frac{\mathrm{d}}{\mathrm{d} p_{2}} F\left(p_{1}, p_{2}\right), \frac{\mathrm{d}}{\mathrm{d} p_{1}} F\left(p_{1}, p_{2}\right)\right)+\frac{\pi}{4}\right) \frac{2}{\pi} \quad(\bmod 1) \cdot 2\right|
$$


Hierbei ist $F\left(p_{1}, p_{2}\right)$ das analytische Modell als Funktion in Abhängigkeit von den untersuchten Parameter $p_{1}$ und $p_{2}$. Mit der Funktion atan2 wird der Winkel zwischen den Gradienten in $p_{1}$ - und $p_{2}$-Richtung bestimmt. Damit können dann

$$
A Z_{1}=\frac{1}{N} \sum_{i=1}^{N} a z
$$

und

$$
A Z_{2}=1-\sqrt{\frac{1}{N} \sum_{i=1}^{N}\left|a z-A Z_{1}\right|^{2}}
$$

berechnet werden. In den Gleichungen (16) und (17) ist $N$ die Anzahl der betrachteten Punkte für die $p_{2}$ berechnet wurde. An den Abbildungen 1 und 2 sollen die Aussagen dieser Kriterien beschrieben werden. In Abb. 1 ist ein Problemfall für den Optimierer zu sehen. Beide Größen $\lambda_{1}$ und $\lambda_{2}$ haben den gleichen Einfluss auf den Ausgangsdruck $p_{2}$. Das ist am Qualitätskriterium $A Z_{1}$, dessen Wert größser als 0,5 ist, abzulesen. Dass der Zusammenhang linear ist, kann am zweiten Qualitätskriterium $A Z_{2}$, dessen Wert 1 ist, abgelesen werden. Das bedeutet der Optimierer findet Lösungen, bei denen $\alpha \lambda_{1}+\lambda_{2}=$ const. gilt, und kann auch mit mehreren Arbeitspunkten keine eindeutige Lösung finden. Auch in Abb. 2 haben die Parameter einen direkten Zusammenhang, was am Wert des Qualitätskriteriums $A Z_{1}$ abzulesen ist. Da hier aber in Bereichen kein linearer Zusammenhang besteht, kann mit mehreren Arbeitspunkten eine eindeutige Lösung gefunden werden. Dies kann am Qualitätskriterium $A Z_{2}$ abgelesen werden.

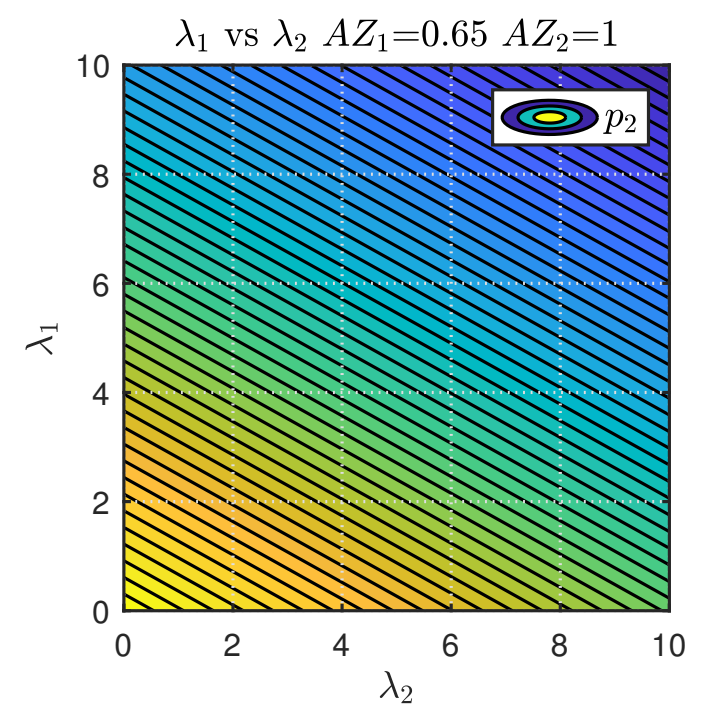

Abb. 1: Abhängigkeit des Ausgangsdrucks $p_{2}$ von $\lambda_{1}$ und $\lambda_{2}$

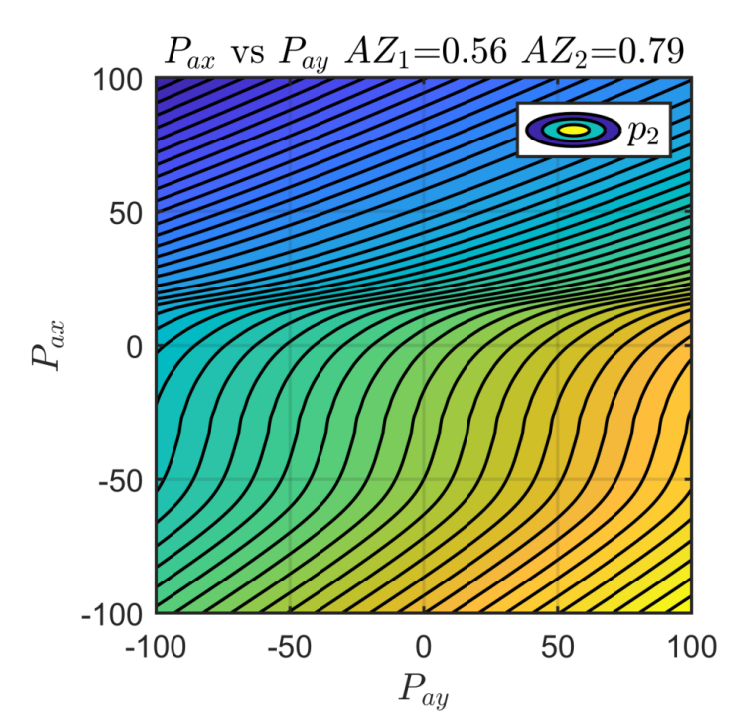

Abb. 2: Abhängigkeit des Ausgangsdrucks $p_{2}$ von $P_{a x}$ und $P_{a y}$

\section{Versuchsdurchführung}

\subsection{Fehlerszenarien}

Für die Fehlerszenarien wird zuerst ein Punkt festgelegt, für den in allen Szenarien optimiert wird. Dafür bietet sich der Referenz-Arbeitspunkt $A P_{0}$ im Verdichterkennfeld an, da bei diesem die Werte für Ein- und Ausgangsdruck, Temperatur, Drehzahl und Massenfluss gegeben sind. Es werden Szenarien erstellt, in denen echte Fehler simuliert werden, und solche, in denen Fehler die Robustheit des Optimierers testen. Alle Szenarien haben folgende Eigenschaften gemeinsam: 
- Es werden zehn Wiederholungen und dann eine Mittelung der Ergebnisse durchgeführt.

- Auf die Messgrößen werden 1\% Rauschen addiert.

- Alle Szenarien werden mit 5, 10 und 15 Arbeitspunkten ausgeführt, um den Effekt der Anzahl der Arbeitspunkte zu bestimmen.

Das wichtigste Szenario ist die Variation der Optimierungsvariablen (Parameter). Diese werden mit einem gleichverteilten Rauschen

$$
P_{R}=P+a Z
$$

beaufschlagt. Hierbei ist $P_{R}$ der verrauschte Parameter, $P$ der Originalparameter, $Z$ der Wert einer gleichverteilten Zufallszahl im Interval $[-1,1]$, $a$ ein Skalierungsparameter zur Einstellung der SignalRausch-Verhältnisses.

Das zweite Szenario untersucht den Einfluss von Rauschen auf die Messgrößen, auch hier wird die Gleichung (18) verwendet. Im dritten Szenario werden die Startwerte für den Optimierer variiert. Im vierten Szenario werden fehlerhafte Messstellen für die Eingangsgrößen simuliert. Im fünften Szenario werden die Messwerte zunächst auf Plausiblität geprüft und Messwerte fehlerhafter Messstellen eliminiert. Diese Plausibilitätsprüfung funktioniert nach folgendem Prinzip: Die Eingangsmesswerte werden in die Gleichungen (1) und (5) eingesetzt und die berechneten Werte werden mit den Ausgangsmesswerten verglichen. Wenn diese um $25 \%$ oder mehr abweichen, wird dieser Arbeitspunkt ignoriert.

\subsection{Fehlermetrik}

Um die Qualität der Optimierer bzw. die gefundenen Optima zu vergleichen, wird eine Fehlermetrik $F M$ eingeführt. Dabei wird der Restfehler nach der Optimierung auf den eingeführten Fehler $F_{s z}$ im Szenario mit

$$
F M=\min _{p} \sum_{i}\left(F_{i}\left(\hat{x}_{i}, p\right)-\hat{y}_{i}\right)^{2} \cdot F_{s z}^{-2}
$$

normiert. Die Größe $F_{s z}$ beschreibt den zu korrigierenden Fehler im Szenario und wird zur Normierung verwendet. Damit ergeben sich vier Bereiche für $F M$

- $F M>1$ : Lösung des Optimierer ist schlechter als Startwert.

- $F M=1:$ Lösung des Optimierer entspricht dem Startwert.

- $F M<1$ : Lösung des Optimierer ist besser als Startwert.

- $F M=0$ : Lösung des Optimierer entspricht der Veränderung des Originalzustands.

Damit werden die Optimierer in verschiedenen Fehlerszenarien miteinander vergleichbar.

\subsection{Versuchsablauf}

Die Versuche werden in Matlab durchgeführt. Daher wird das Modell mit den Gleichungen (1), (5) und (6) in Matlab umgesetzt und ein Kennfeld eingelesen. Ausgewählt wurde ein Standardkennfeld, siehe Abb. 3, und wichtige Punkte ausgelesen. Die wesentlichen Größen und Parameter sind:

- Referenz-Arbeitspunkt: $A P_{0}$ mit $p_{1}=5,9$ bar; $T_{1}=20^{\circ} \mathrm{C} ; \dot{m}=10,6 \frac{\mathrm{kg}}{\mathrm{s}} ; \dot{V}=136 \frac{\mathrm{kJ}}{\mathrm{kg}} ; p_{2}=15 \mathrm{bar} ; T_{2}=168{ }^{\circ} \mathrm{C}$

- Drehzahlkennlinien in $N_{N}=N / N_{0}$ mit $N=142101 / \min$ und $N_{N}=(0,7 ; 0,8 ; 0,9 ; 1,0 ; 1,05)$

- verwendetes Gas: Stickstoff; Kompressibilitätsfaktor $Z=1$, spezifische Gaskonstante $R_{S}=296,8 \frac{\mathrm{J}}{\mathrm{kg} \mathrm{K}}$

Für die Approximation werden dann die Bildpunkte der x-Achse und der y-Achse über die Eckpunkte des Rahmen in den Volumenstrom $\dot{V}$ und die polytrope Förderhöhe $Y_{P}$ umgerechnet.

Für die Versuchsreihen werden mit dem Modell für die festgelegten Eingangsdaten $p_{1}, T_{1}$ und $\dot{m}$ die Ausgangsdaten $p_{2}$ und $T_{2}$ erzeugt. Dabei werden je nach Szenario die Optimierungsvariablen geändert, die Messdaten manipuliert und die Anzahl der Arbeitspunkte variiert. Für die verschieden Arbeitspunkte wird der Massenfluss in gleichen Schritten zwischen $20 \%$ und $100 \%$ im Verhältnis zum 


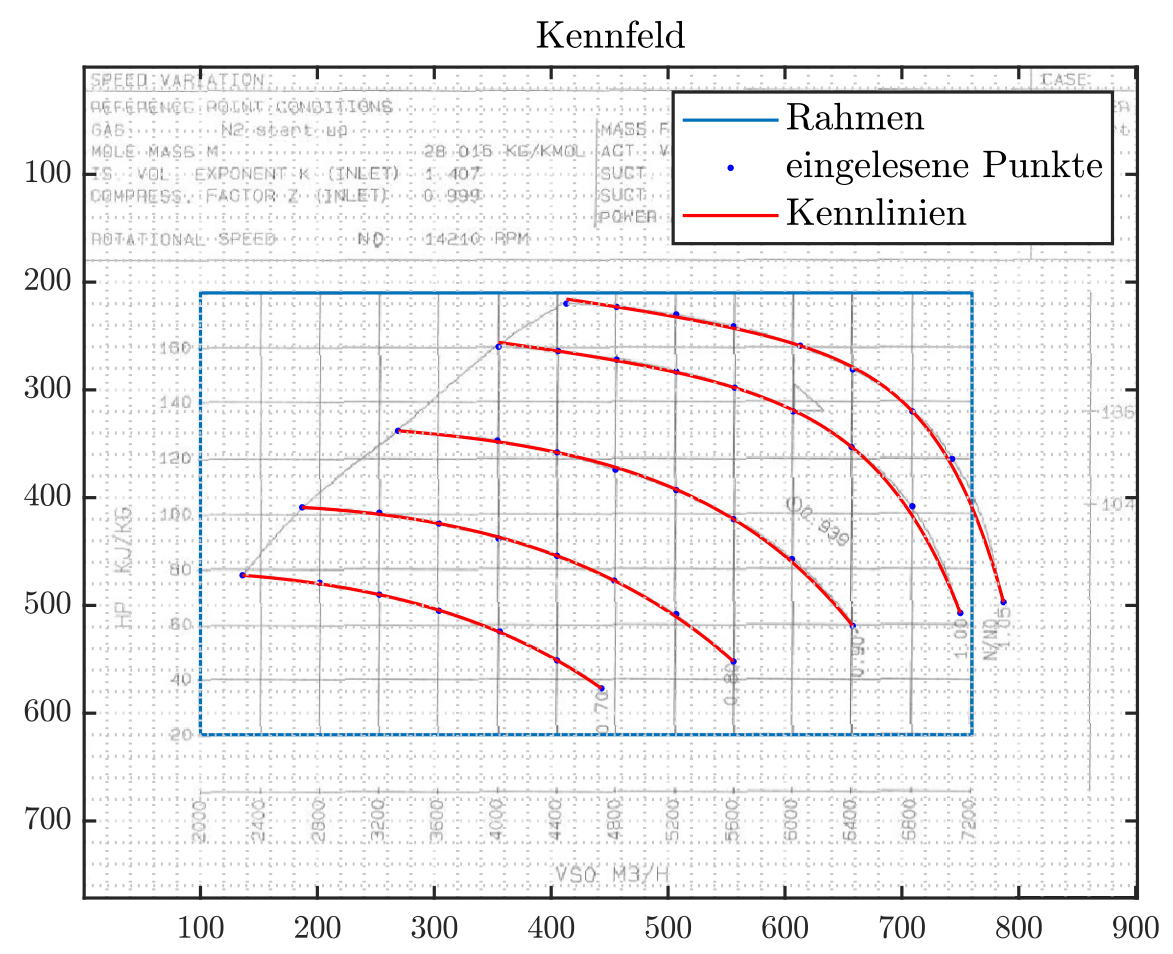

Abb. 3: Kennfeld des Verdichters

Referenz-Arbeitspunkt variiert. Aufgabe der Optimierung ist dann die Änderung der Optimierungsvariablen korrekt zu bestimmen. Die Grundlage dafür bilden die generierten Eingangs- und Ausgangsdaten. Der Startwert für den Optimierer sind die Werte der Optimierungsvariablen aus dem ReferenzArbeitspunkt. Um reale Messdaten zu simulieren, werden diese mit einem Rauschen entsprechend Gleichung (18) beaufschlagt.

Zuerst wird das Fehlerszenario 1 mit der Variation der Modellparameter untersucht. An diesem wird dann mit Hilfe des Qualitätskriteriums eine Einschätzung, welche Optimierungsvariablen zusammen optimiert werden können, vorgenommen. Mit diesen Optimierungsvariablen werden dann alle weiteren Fehlerszenarien durchgespielt und über die Fehlermetrik verglichen. Danach wird der Einfluss auf die Rechenzeit durch die drei unterschiedlichen Kennfeldapproximationen ermittelt. Abschließend wird in einem angepassten Szenario überprüft, ob die erweiterte Zielfunktion (14) für eine robuste Optimierung zu verbesserten Ergebnissen, d. h. einer geringen Abhängigkeit von der Lage der einzelne Arbeitspunkte führt.

\section{Ergebnisse}

Das erste Szenario liefert die Ergebnisse in Abb. 4, dabei werden fünf parameter $\lambda_{1}, \lambda_{2}, N, P_{a x}$ und $P_{a y}$ zur Optimierung ausgewählt. Die anschließende Betrachtung der Qualitätskriterien für die Optimierungsparameter in Tab. 2 und 3 zeigt, dass zwischen $\lambda_{1}$ und $\lambda_{2}$ sowie zwischen $P_{a x}$ und $P_{a y}$ eine Abhängigkeit besteht. Für eine Analyse müssen die dazugehörigen Grafiken analysiert werden (s. Abschnitt 2.6). In Abb. 1 ist eindeutig ein linearer Zusammenhang zwischen den Optimierungsvariablen $\lambda_{1}$ und $\lambda_{2}$ zu sehen. Diese werden daher im folgenden Versuchsverlauf gleichgesetzt. Die Abb. 2 muss eingehender betrachtet werden. Wird der Suchraum auf realistische $25 \%$ bezogen auf den Referenzbetriebspunkt eingeschränkt wird sinkt das Qualitätskriterium $A Z_{1}$ auf 0,56 und $A Z_{2}$ auf 0,77. Die Optimierungsvariablen $P_{a x}$ und $P_{a y}$ können also gemeinsam optimiert werden. Die Ergebnisse aus der Optimierung sind in Abb. 5 zu sehen und zeigen eindeutig eine Verbesserung aller getesteten Optimierungsverfahren. 


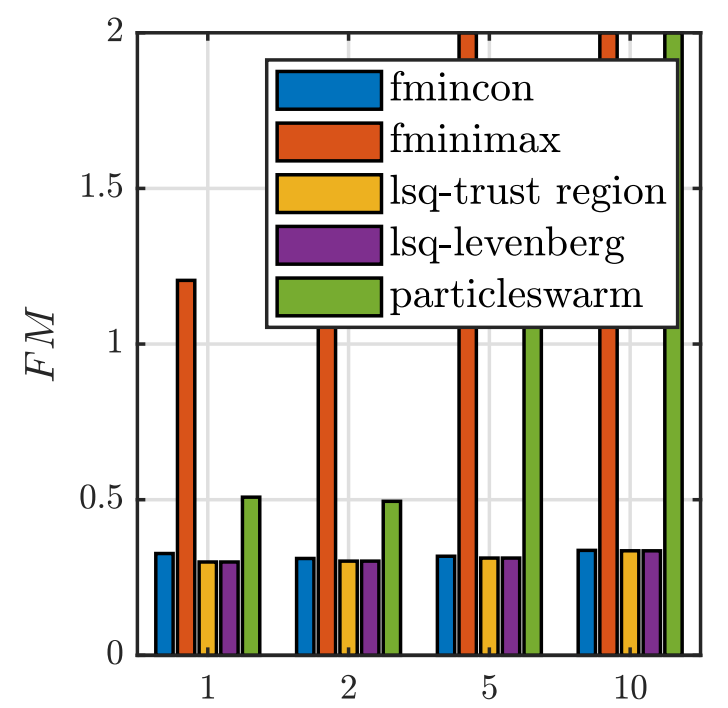

Abw. Optimierungsparameter in \%

Abb. 4: Szenario $1 F M$ mit 5 Freiheitsgraden

Tab. 2: Qualitätskriterium $A Z_{1}$

\begin{tabular}{ccccc}
\hline$A Z_{1}$ & $\lambda_{1}$ & $\lambda_{2}$ & $N$ & $P_{a x}$ \\
\hline \hline$P_{a y}$ & 0.05 & 0.03 & 0.51 & 0.86 \\
$P_{a x}$ & 0.05 & 0.03 & 0.56 & \\
$N$ & 0.06 & 0.03 & & \\
$\lambda_{2}$ & 0.65 & & & \\
\hline
\end{tabular}

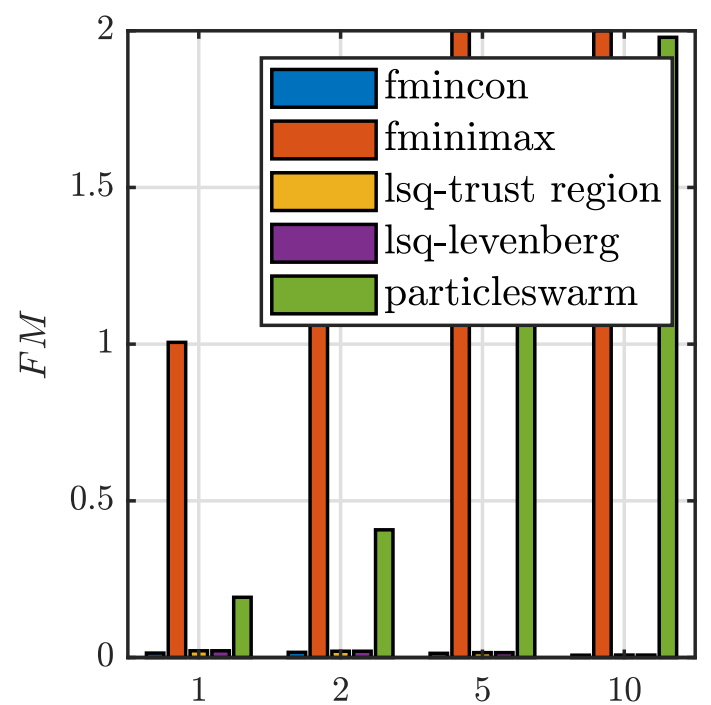

Abw. Optimierungsparameter in \%

Abb. 5: Szenario $1 F M$ mit 4 Freiheitsgraden

Tab. 3: Qualitätskriterium $A Z_{2}$

\begin{tabular}{ccccc}
\hline$A Z_{2}$ & $\lambda_{1}$ & $\lambda_{2}$ & $N$ & $P_{a x}$ \\
\hline \hline$P_{a y}$ & 0.98 & 1 & 0.79 & 0.79 \\
$P_{a x}$ & 0.93 & 0.96 & 0.82 & \\
$N$ & 0.98 & 1 & & \\
$\lambda_{2}$ & 1 & & & \\
\hline
\end{tabular}

Für die folgenden Versuche wird auf fmincon und den Partikelschwarmoptimierer auf Grund des schlechten Abschneidens verzichtet. Weiterhin werden nur noch vier Optimierungsvariablen $\lambda_{1}=\lambda_{2}=\lambda, N, P_{a x}$ und $P_{a y}$ verwendet.

Bei den Szenarien zum Einfluss von Rauschen in Abb. 6 und Startpunktänderung Abb. 7 liefern alle drei Optimierer ähnliche Ergebnisse. Beim Levenberg-Marquardt-Algorithmus macht sich die fehlende Grenze des Suchraumes durch ein schlechteres Ergebnis von $F M$ bemerkbar.

Die Grenzen der Optimierung sind bei der Betrachtung von Szenario 4 in Abb. 8 zur Untersuchung des Einflusses von fehlerhaften Messstellen zu sehen. Bei Einführung auch nur einer falschen Messstelle, hier definiert als Abweichung von größer $25 \%$ vom wahrem Wert, ist keine erfolgreiche Optimierung möglich. Um eine erfolgreiche Optimierung zu ermöglichen, müssen wie in Szenario 5, siehe Abb. 9, eine Plausibilitätsprüfung vor der eigentlichen Optimierung vorgenommen und stark fehlerhafte Messstellen ausgeschlossen werden.

Für die Untersuchung zum Geschwindigkeitseinfluss der Kennfeldapproximation wird das durch die Dimensionsanalyse geänderte Kennfeld in Abb. 10 gezeigt. Es ist deutlich die Verkleinerung des Bereiches zu erkennen, in dem approximiert werden muss. Dies zeigt sich auch nach der Geschwindigkeitsanalyse, indem Szenario 1 hundert Mal wiederholt wird. Dabei werden alle drei Kennfeldapproximationen bei der Optimierung durch den Trust-region-reflective-Algorithmus auf Basis der benötigten Zeit verglichen, siehe Tab. 4.

Die Ergebnisse zur Untersuchung der gewichteten Zielfunktion sind in Abb. 11 zu sehen. Es fällt auf, dass der einzelne Arbeitspunkt auf der linken Seite besser, die vier gruppierten Arbeitspunkte auf der rechten Seite aber etwas schlechter optimiert sind. Dies bedeutet, dass abgewogen werden muss, ob die Zielfunktion (14) verwendet wird, denn wenn der einzelne Arbeitspunkt stark fehleranfällig ist, wiegt dies die schlechtere Optimierung der gruppierten Arbeitspunkte nicht auf. 


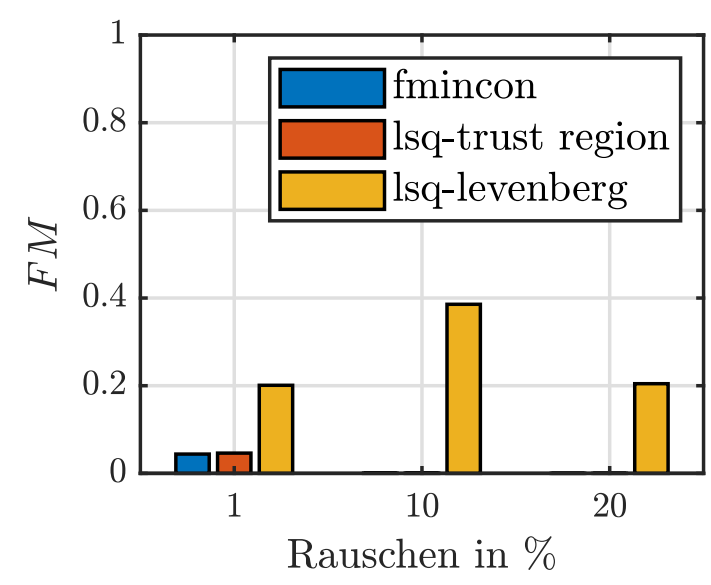

Abb. 6: Szenario 2

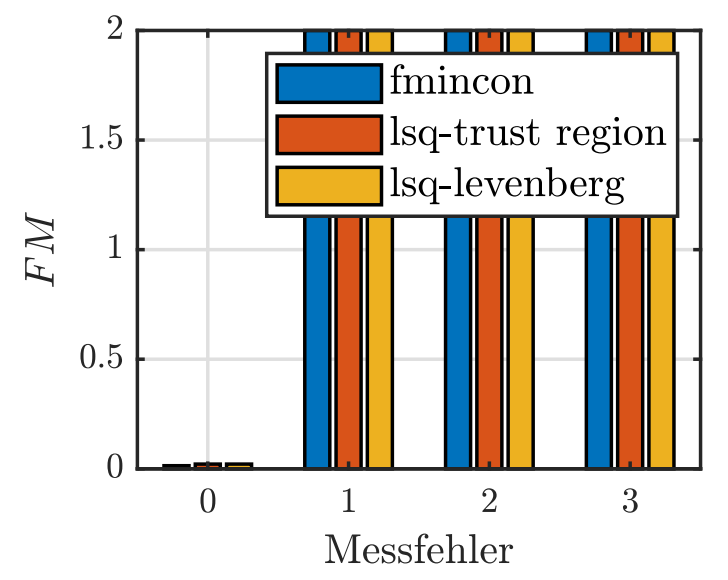

Abb. 8: Szenario 4 - Messfehler

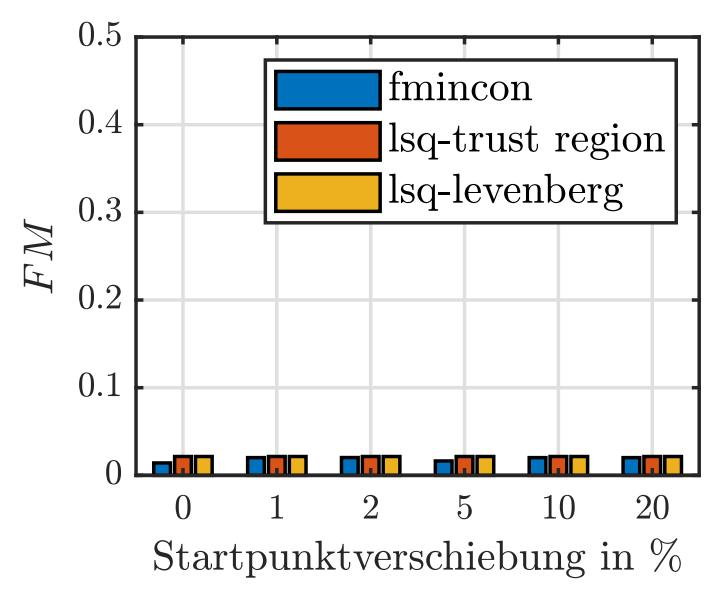

Abb. 7: Szenario 3

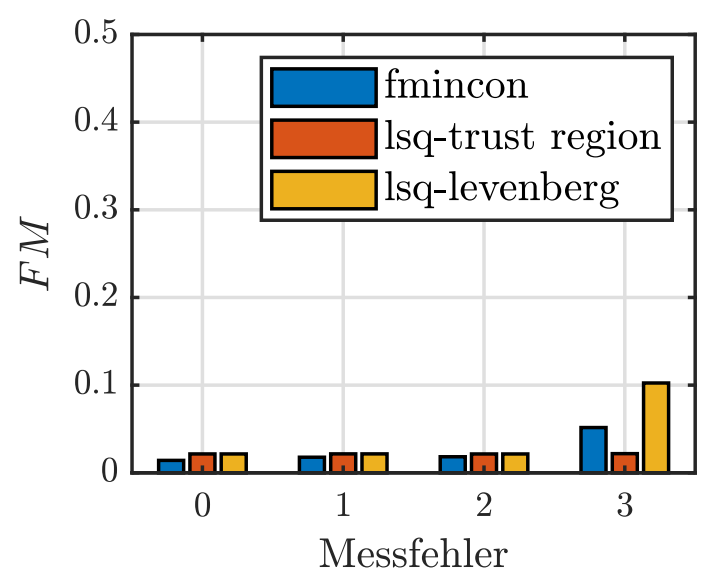

Abb. 9: Szenario 5 mit Plausibilitätsprüfung

\section{Auswertung}

Das Ergebnis von Szenario 1 mit 5 Freiheitsgraden, siehe Abb. 4, zeigt deutlich, dass eine Vorauswahl der gemeinsam zu optimierenden Parameter notwendig ist. Erst die richtige Bewertung und Anpassung der Optimierungsvariablen mit Hilfe der Gütekriterien $A Z_{1}$ und $A Z_{2}$ ermöglicht eine Optimierung mit minimalem Restfehler. Nach der Wiederholung von Szenario 1 mit 4 Freiheitsgraden ist es möglich, eine erste Bewertung der verwendeten Optimierungsalgorithmen vorzunehmen. Der Optimierungsalgorithmus fmincon und der Partikelschwarmoptimierer sind dabei als ungeeignet einzuschätzen. Die Verwendung von Partikelschwarmoptimierern erfordert zudem eine um den Faktor 10 längere Rechenzeit im Vergleich zu den anderen Optimierungsalgorithmen. Die weiteren vier Szenarien zeigen bei den verbliebenen drei Optimierungsalgorithmen ähnliche Ergebnisse. Hierbei ist allerdings beim Verfahren lsqcurvefit der Trust-region-reflective-Algorithmus gegenüber dem Levenbergmarquardt-Algorithms zu bevorzugen.

Tab. 4: Zeitdauer von jeweils 100 Optimierungen bei Variation der Kennfeldapproximation

\begin{tabular}{cc}
\hline Methode & Zeit \\
\hline \hline Exponentialfunktion mit Interpolation & $4,23 \mathrm{~s}$ \\
Polynom dritter Ordnung & $20,52 \mathrm{~s}$ \\
Dimensionsanalyse mit Polynom dritter Ordnung & $10,79 \mathrm{~s}$ \\
\hline
\end{tabular}




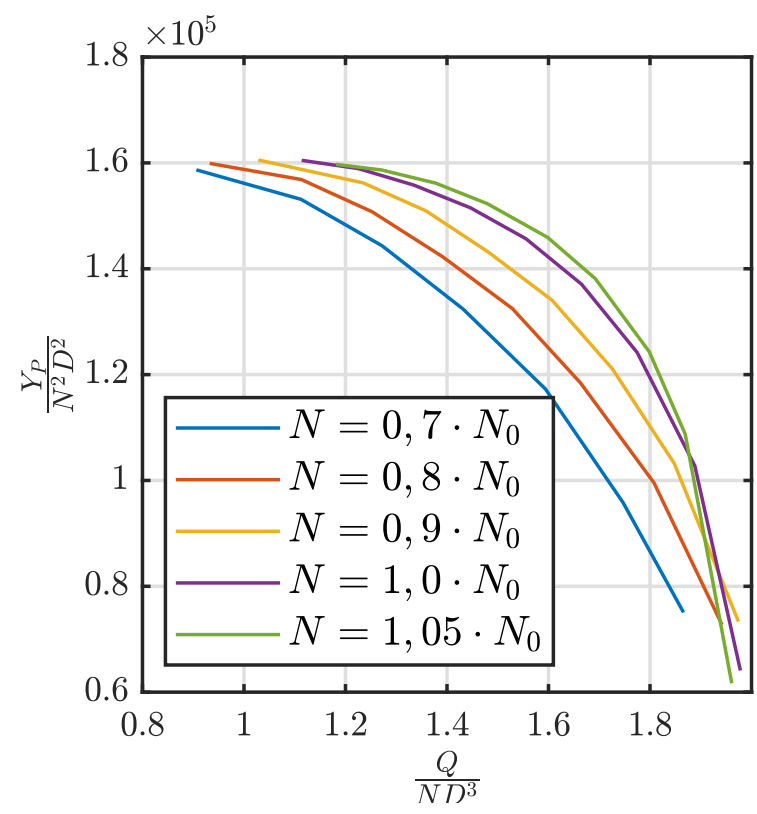

Abb. 10: Kennfeld nach der Dimensionsanalyse

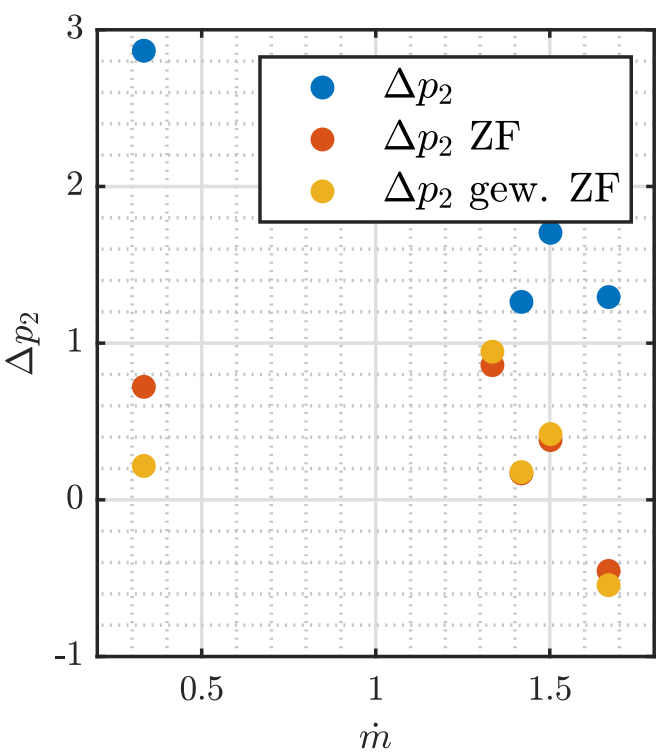

Abb. 11: Vergleich zwischen der Standard- und der gewichteten Zielfunktion

\section{Zusammenfassung}

Der Beitrag befasst sich mit der vergleichenden Analyse der Identifikation der Parameter von Simulationsmodellen auf Basis unterschiedlicher numerischer Optimierungsverfahren. Neben gradientenbasierten Verfahren wird auch ein Partikelschwarmoptimierer betrachtet. Die parameteridentifikation wird mit Werten der relevanten Modellgrößen an verschiedenen Arbeitspunkten durchgeführt. Als wichtiges Problem stellt sich die Identifizierbarkeit der Parameter dar. Hierfür werden zwei Kriterien eingeführt, die eine numerische Analyse erlauben. Die Optimierungsverfahren werden in verschiedenen Szenarien untersucht und miteinander verglichen. Hierzu gehören unterschiedlich Signal-RauschVerhältnisse, verschiedene Startwerte für die Optimierungsvariablen sowie stark fehlerbehafte Messwerte bei den Eingangsgrößen. Im Ergebnis können die in Matlab implementierten Verfahren fmincon und lsqcurvefit mit dem Trust-region-refective-Algorithmus empfohlen werden.

\section{Förderung}

Dieses Projekt wurde vom BMWi mit der Nummer ZF4088617GR9 gefördert.

\section{Literatur}

[1] Schäffler, S. Globale Optimierung.Springer-Verlag Berlin Heidelberg, 2014.

[2] Ulbrich, M.; Ulbrich, S. Nichtlineare Optimierung. Birkhäuser Basel, 2012.

[3] Flockermann, D. Einsatz von Optimierungsverfahren zur Bestimmung der Parameter nichtlinearer Ersatzschaltbilder von elektrischen Maschinen. Dortmund, 2003.

[4] Wernholt, E.; Gunnarsson, S. Nonlinear Identification of a Physically Parameterized Robot Model. 14th IFAC Symposium on System Identification, Newcastle, Australia, 2006.

[5] Michalewicz, Z.; Schmidt, M. Evolutionary Algorithms and Constrained Optimization. Springer, Boston, MA, 2003.

[6] Bäck, T., Schwefel, H. An Overview of Evolutionary Algorithms for Parameter Optimization. Evolutionary computation. 1993, Bd. 1(1).

[7] Thomas, P. Simulation of industrial processes for control engineers 1999, S. 239-255

[8] Wright, M.H. The interior-point revolution in optimization: History, recent developments, and lasting consequences. Bull. Amer. Math. Soc. 42 (2005), 39-56

[9] Gröll, L. Methodik zur Integration von Vorwissen in die Modellbildung. KIT Scientific Publishing, 2015.

[10] E. Buckingham: The principle of similitude. In: Nature. 96, 1915, S. 396-397 